\title{
Multisoftware Reproducibility Study of Stress and Rest Myocardial Blood Flow Assessed with 3D Dynamic PET/CT and a 1-Tissue-Compartment Model of ${ }^{82} \mathrm{Rb}$ Kinetics
}

\author{
Robert A. deKemp ${ }^{1}$, Jerome Declerck ${ }^{2}$, Ran Klein ${ }^{1}$, Xiao-Bo Pan², Ryo Nakazato ${ }^{3}$, Christine Tonge ${ }^{4}$, \\ Parthiban Arumugam ${ }^{4}$, Daniel S. Berman ${ }^{3}$, Guido Germano ${ }^{3}$, Rob S. Beanlands ${ }^{1}$, and Piotr J. Slomka ${ }^{3}$ \\ ${ }^{1}$ University of Ottawa Heart Institute, National Cardiac PET Centre, Ottawa, Canada; ${ }^{2}$ Siemens Healthcare Molecular Imaging, \\ Oxford, United Kingdom; ${ }^{3}$ Cedars-Sinai Medical Center, Los Angeles, California; and ${ }^{4}$ Central Manchester University Hospitals, \\ NHS Foundation Trust, Manchester, United Kingdom
}

Routine quantification of myocardial blood flow (MBF) requires robust and reproducible processing of dynamic image series. The goal of this study was to evaluate the reproducibility of 3 highly automated software programs commonly used for absolute MBF and flow reserve (stress/rest MBF) assessment with ${ }^{82} \mathrm{Rb}$ PET imaging. Methods: Dynamic rest and stress ${ }^{82} \mathrm{Rb}$ PET scans were selected in 30 sequential patient studies performed at 3 separate institutions using 3 different 3-dimensional PET/CT scanners. All 90 scans were processed with 3 different MBF quantification programs, using the same 1-tissue-compartment model. Global (left ventricle) and regional (left anterior descending, left circumflex, and right coronary arteries) MBF and flow reserve were compared among programs using correlation and BlandAltman analyses. Results: All scans were processed successfully by the 3 programs, with minimal operator interactions. Global and regional correlations of MBF and flow reserve all had an $R^{2}$ of at least 0.92 . There was no significant difference in flow values at rest $(P=0.68)$, stress $(P=0.14)$, or reserve $(P=0.35)$ among the 3 programs. Bland-Altman coefficients of reproducibility $(1.96 \times$ SD) averaged 0.26 for MBF and 0.29 for flow reserve differences among programs. Average pairwise differences were all less than $10 \%$, indicating good reproducibility for MBF quantification. Global and regional SD from the line of perfect agreement averaged 0.15 and $0.17 \mathrm{~mL} / \mathrm{min} / \mathrm{g}$, respectively, for MBF, compared with 0.22 and 0.26 , respectively, for flow reserve. Conclusion: The 1-tissue-compartment model of ${ }^{82} \mathrm{Rb}$ tracer kinetics is a reproducible method for quantification of MBF and flow reserve with 3-dimensional PET/CT imaging.

Key Words: myocardial blood flow quantification; myocardial perfusion imaging; rubidium positron emission tomography; coronary flow reserve; myocardial perfusion reserve

J Nucl Med 2013; 54:571-577

DOI: 10.2967/jnumed.112.112219

\footnotetext{
Received Aug. 15, 2012; revision accepted Nov. 5, 2012.

For correspondence or reprints contact: Robert A. deKemp, Cardiac Imaging, University of Ottawa Heart Institute, 40 Ruskin St., Ottawa, Ontario, K1Y 4W7, Canada.

E-mail: RAdeKemp@ottawaheart.ca

Published online Feb. 27, 2013.

COPYRIGHT (C 2013 by the Society of Nuclear Medicine and Molecular Imaging, Inc.
}

A bsolute quantification of myocardial blood flow $(\mathrm{MBF})$ at stress and rest with dynamic PET imaging is an important tool for clinicians and provides information complementary to relative myocardial perfusion imaging (1-3). With standard list-mode acquisition and fast image reconstruction, dynamic, gated, and standard static perfusion images can be obtained with a single injection of the radiopharmaceutical and without additional imaging time. Automated image analysis tools are required for reliable and robust clinical use of dynamic data for MBF quantification (4). The performance of several such software programs for MBF quantification has been reported recently (5-9), each of which uses different tracers and methods of segmenting and sampling the left ventricular myocardium and blood-pool activity to obtain input curves. Although each of these tools greatly simplifies MBF quantification, uses the same tracer kinetic model for ${ }^{82} \mathrm{Rb}$-rubidium (10), and has been validated individually, the effect of different model implementations has not been characterized. Previous studies have compared different tracer kinetic models and implementations for ${ }^{13} \mathrm{~N}$ ammonia $(11,12)$ but not for ${ }^{82} \mathrm{Rb}$-rubidium and in particular not for 3-dimensional (3D)-mode PET, which is the current standard technology. We aimed to compare 3 software packages that have been implemented clinically for the quantitative analysis of MBF and myocardial flow reserve (MFR) with rubidium PET. These methods include QPET (CedarsSinai) (5), syngoMBF (Siemens Healthcare) (6), and FlowQuant (University of Ottawa Heart Institute) (7). These 3 software programs perform MBF quantification using different implementations of the same 1-tissue-compartment model and different methods of segmenting the left ventricle and sampling tracer activity in the myocardium and blood pool.

\section{MATERIALS AND METHODS}

\section{Study Design}

Ninety patients were included in the study. All were referred clinically for the evaluation of known or suspected ischemic heart disease using rest-stress myocardial perfusion imaging with ${ }^{82} \mathrm{Rb}$ PET ( $n=30$ sequential studies per site: June 2009 at Ottawa 
Heart, December 2009 to March 2010 at Cedars-Sinai, May to October 2010 at Central Manchester). After the initial quality assurance review, 1 scan from Central Manchester with an extreme motion artifact was replaced with a patient from the same period to maintain a balanced design. The population sample is representative of patients commonly referred for diagnosis of coronary artery disease and evaluation of myocardial ischemia, including patients with a previous history of myocardial infarction or revascularization. All images (90 rest and 90 stress) were processed using all 3 software packages at the respective development sites, to obtain MBF and MFR for the entire myocardial region (global) and regionally in the 3 main coronary artery territories according to the guidelines of the American Heart Association on polar map segmentation (13). The global and regional MBF and MFR were then exchanged among centers for analysis. The study protocols were approved by each of the institutional human research ethics boards. All patients gave signed informed consent.

\section{Image Acquisition}

The ${ }^{82} \mathrm{Rb}$ PET studies were performed at 3 sites, with 3 types of scanners from 2 different vendors, using slightly different imaging and reconstruction protocols and 2 types of ${ }^{82} \mathrm{Rb}$ generators, according to the routine clinical practice at each center as described below.

Ottawa Heart Institute. Scans were acquired on a Discovery RX PET-VCT 64-slice scanner (GE Healthcare) using a 3D list-mode acquisition after a 30-s square-wave intravenous infusion (14) of ${ }^{82} \mathrm{Rb}$ (10 MBq/kg; Jubilant DraxImage). Patients were studied after an overnight fast and were instructed to refrain from caffeine- or theophylline-containing products or medications for $12 \mathrm{~h}$ before the study. After a normal end-expiration low-dose, fast $(0.4 \mathrm{mSv}$, $120 \mathrm{kVp}, 20-200 \mathrm{~mA}$ [automatically modulated using a 50\% noise index], $1.5 \mathrm{~s}$ ) CT acquisition for attenuation correction (AC), ${ }^{82} \mathrm{Rb}$ was administered to the patient and a 6-min PET acquisition was started when the increase in true counts exceeded $10 \mathrm{kcps}$. Cardiac stress was induced using dipyridamole $(0.142 \mathrm{mg} / \mathrm{min})$ administered over $5 \mathrm{~min}$, and a second ${ }^{82} \mathrm{Rb}$ infusion and PET acquisition were started $3 \mathrm{~min}$ later. Aminophylline was injected $4 \mathrm{~min}$ later to reduce side effects in all patients. A second CT scan was obtained for AC of the stress PET scan. During the entire examination, the patient was instructed to breathe normally. CT AC alignment with PET was verified visually by an experienced technologist and corrected if necessary by manual 3D translation, using the vendor ACQC program. Images were reconstructed using filtered backprojection and a 12-mm Hann filter into 14 time frames $(9 \times 10 \mathrm{~s}$, $3 \times 30 \mathrm{~s}, 1 \times 60 \mathrm{~s}$, and $1 \times 120 \mathrm{~s}$; total, $6 \mathrm{~min}$ ).

Cedars-Sinai Medical Center. Patients were scanned on a Biograph True Point PET/CT 64-slice system (Siemens Healthcare) in 3D mode. Patients were studied after an overnight fast and were instructed to refrain from caffeine- or theophylline-containing medications for $24 \mathrm{~h}$ before the study. Two CT-based transmission scans $(0.4 \mathrm{mSv} ; 120 \mathrm{kVp}$; effective tube current, $26 \mathrm{~mA}$ [11-mAs quality reference]; $3.3 \mathrm{~s}$ ) were obtained before the rest study and after the stress study for AC. Rest and stress CT transmission scans were acquired at end-expiration breath-hold. The CT attenuation map registration with the PET images was verified visually by an experienced technologist, and alignment was corrected if necessary by manual 3D translation. Regional myocardial perfusion was first assessed during rest using $925-1,850 \mathrm{MBq}$ of ${ }^{82} \mathrm{Rb}$ (Bracco Diagnostics) infused intravenously at $35-50 \mathrm{~mL} / \mathrm{min}$. A 6-min rest scan was started simultaneously with the start of the rest infusion. Immediately after completion of rest imaging, a second stress CT transmission scan was obtained. Two minutes after the start of adenosine infusion $(0.14 \mathrm{mg} / \mathrm{kg} / \mathrm{min}$ for $7 \mathrm{~min})$, 925-1,850 MBq of ${ }^{82} \mathrm{Rb}$ were administered using a separate intravenous line in the other arm to prevent interference with the adenosine infusion. Sixteen dynamic frames were reconstructed $(12 \times 10 \mathrm{~s}, 2 \times 30 \mathrm{~s}, 1 \times 60 \mathrm{~s}$, and $1 \times 120 \mathrm{~s}$; total, $6 \mathrm{~min})$ using the vendor iterative method (Fourier rebinning + 2-dimensional attenuation-weighted ordered-subsets expectation maximization) with 2 iterations, 8 subsets, and 8 -mm gaussian postprocessing filter.

Central Manchester University Hospitals. Patients underwent serial rest-stress imaging on a Biograph mCT 64-slice scanner (Siemens Healthcare) using list-mode 3D acquisition after ${ }^{82} \mathrm{Rb}$ infusion (Bracco Diagnostics). Patients were instructed to abstain from caffeine for $12 \mathrm{~h}$ before imaging. Low-dose CT $(0.4 \mathrm{mSv}$; $120 \mathrm{kVp}$; effective tube current, $26 \mathrm{~mA}$ [11-mAs quality reference]; $3.3 \mathrm{~s}$ ) was performed for $\mathrm{AC}$, during normal breathing. A $1,480-\mathrm{MBq}(40 \mathrm{mCi})$ infusion of ${ }^{82} \mathrm{Rb}$ was administered intravenously at $50 \mathrm{~mL} / \mathrm{min}$. Acquisition started with the tracer infusion and continued for $6 \mathrm{~min}$. Stress was induced with adenosine infused at $140 \mathrm{cg} / \mathrm{kg} / \mathrm{min}$ for $4.5 \mathrm{~min}$, with acquisition starting 2.5 min later. Alignment between non-attenuation-corrected PET and CT images was verified and corrected manually by an experienced technologist for any patient body motion. In cases of significant patient motion between PET and CT, an additional lowdose CT scan was acquired at the end of the study for stress AC. Both rest and stress dynamic images were reconstructed into 26 time frames $(12 \times 5 \mathrm{~s}, 6 \times 10 \mathrm{~s}, 4 \times 20 \mathrm{~s}$, and $4 \times 40 \mathrm{~s}$; total, $6 \mathrm{~min})$ using the vendor standard ordered-subsets expectation maximization 3D reconstruction (2 iterations, 24 subsets) with $6.5-\mathrm{mm}$ gaussian postprocessing filter.

\section{MBF Analysis}

Global and regional MBF and MFR were obtained using 3 highly automated software programs listed in Table 1 and illustrated in Supplemental Figure 1 (supplemental materials are available online only at http://jnm.snmjournals.org). The analysis of all studies was performed independently by 3 different expert operators (1 for each software tool) who did not know the results obtained by the other methods.

syngoMBF. For syngoMBF, each case was processed automatically with a quality control step in which the operator confirmed or modified the results of automatic reorientation $(15,16)$ and invoked an additional option for advanced motion correction (17) if needed. The myocardium was segmented automatically and sampled into 15 rings with 36 segments. Myocardial tissue time-activity curves were obtained at each time frame as the averaged value of a region with approximately $1-\mathrm{cm}$ radial thickness around the midmyocardial surface along the radial line. The arterial blood input function used for kinetic analyses was obtained from the dynamic sequence by averaging the activity in a $2-\mathrm{cm}$ cylindric region of interest $1.5 \mathrm{~cm}$ in diameter placed automatically in the middle of the left ventricle in the basal region.

FlowQuant. For FlowQuant, a batch processing study was configured with all the image files and processed automatically without any operator interaction. FlowQuant rest-stress MBF analysis was performed as reported previously (7). Briefly, transaxial dynamic images were automatically reoriented to short-axis slices by fitting partial ellipses to the myocardium in 3 orthogonal planes. A spline model of the left ventricular (LV) myocardium was fit to the short-axis images averaged from 2 to $6 \mathrm{~min}$. On the 
TABLE 1

MBF Quantification Software Programs

\begin{tabular}{|c|c|c|c|c|c|c|c|}
\hline Name & Vendor & $\begin{array}{l}\text { Operating } \\
\text { system }\end{array}$ & $\begin{array}{l}\text { FDA/CE } \\
\text { approval }\end{array}$ & $\begin{array}{l}\text { Blood region } \\
\text { location }\end{array}$ & $\begin{array}{l}\text { Blood region } \\
\text { shape:size }\end{array}$ & $\begin{array}{l}\text { Myocardial region } \\
\text { width }\end{array}$ & $\begin{array}{l}\text { Motion } \\
\text { correction }\end{array}$ \\
\hline FlowQuant & $\begin{array}{c}\text { Ottawa } \\
\text { Heart }\end{array}$ & Windows/Linux & $\begin{array}{l}\text { Both in } \\
\text { progress }\end{array}$ & $\begin{array}{l}\text { LV cavity, } \\
\text { base, } \\
\text { atrium }\end{array}$ & $\begin{array}{l}\text { Cylinder:8-mm } \\
\text { diameter; } \\
\sim 40 \text {-mm } \\
\text { length }\end{array}$ & $4 \mathrm{~mm}$ & Optional \\
\hline syngoMBF & Siemens & Windows & Yes/yes & $\begin{array}{l}\text { LV basal } \\
\text { planes }\end{array}$ & $\begin{array}{l}\text { Cylinder:15-mm } \\
\text { diameter; } \\
\text { 20-mm } \\
\text { length }\end{array}$ & $10 \mathrm{~mm}$ & Yes \\
\hline QPET & $\begin{array}{l}\text { Cedars- } \\
\text { Sinai }\end{array}$ & Windows & Yes/yes & $\begin{array}{l}\text { LV basal } \\
\text { planes }\end{array}$ & $\begin{array}{l}\text { Cylinder:10-mm } \\
\text { diameter; } \\
\text { 20-mm } \\
\text { length }\end{array}$ & $10 \mathrm{~mm}$ & Optional \\
\hline
\end{tabular}

FDA/CE $=$ Food and Drug Administration/CE Mark of the European Commission.

basis of the spline contours, a $0.8-\mathrm{cm}$ by approximately $4-\mathrm{cm}$ cylindric blood region of interest was positioned automatically in the LV and left atrium cavities, maximizing the distance from the myocardial wall and reducing myocardium-to-blood spillover contamination of the arterial input function. Sixteen rings with 36 segments were sampled with 4-mm thickness in the LV myocardium to generate a full polar map; redundancy in the apex was removed, resulting in 496 myocardial regions of interest roughly equal in area. The arterial blood and myocardium regions of interest were used to sample the full dynamic image sequence over $6 \mathrm{~min}$.

QPET. For QPET, the cases were processed in batch mode. LV contours were positioned automatically with a recently described improved algorithm (18). Briefly, the LV contour was determined from the summed dynamic image data skipping the first $2 \mathrm{~min}-$ a method that is based on the original quantitative gated SPECT contour detection principles (19) and the improved valve-plane definition of AC-corrected high-resolution PET. The 3D cylindric region for the LV input function was automatically placed in the middle of the valve plane, with a 1 - by 2 -cm length oriented along the long axis of the heart. The dynamic myocardial samples were obtained from the polar map by analyzing all time frames within the fixed LV contour boundaries as reported for ${ }^{13} \mathrm{~N}$-ammonia MBF (5).

Tracer Kinetics and Flow Reserve. A standard 1-tissuecompartment model (20) was used by all 3 programs, including regional uptake and clearance parameters $\left(K_{1}[\mathrm{~mL} / \mathrm{min} / \mathrm{g}]\right.$ and $k_{2}$ $\left.\left[\min ^{-1}\right]\right)$, blood-to-myocardium spillover fraction $\mathrm{f}_{\mathrm{b}}$, and myocardial partial-volume corrections $\left(1-\mathrm{f}_{\mathrm{b}}\right)$. A Renkin and Crone function $(21,22)$ describing the ${ }^{82} \mathrm{Rb}$ extraction fraction was used to estimate MBF from $K_{1}$, based on the ${ }^{13} \mathrm{~N}$-ammonia correlation reported in humans by Lortie et al. (10).

Stress and rest MBF were computed for each sample in the polar map. MFR was computed by dividing the stress polar map by the rest values at each point. MBF in each vascular territory was then obtained by averaging the polar map segments in the regions of the left anterior descending artery, left circumflex coronary artery, and right coronary artery according to the standard 17-segment American Heart Association model (13).

The following program-specific variations were noted. To reduce noise in the time-activity curves, QPET computations were performed in 70 myocardial regions with equal surface areas. These MBFs were then interpolated using a surface-area-weighted bicubic method to determine MBFs for each of the polar map samples. The global values were computed within the whole LV region bounded by the valve plane. FlowQuant computations were performed in every fourth polar map sector (124 total), and the results were interpolated back to the original polar map dimension before calculation of LV regional and global (polar map mean) MBFs. In syngoMBF, kinetic model fitting was performed on each of the 505 polar map sectors to compute regional MBFs.

\section{Statistical Analysis}

Differences in population means were evaluated using 1-way ANOVA. Agreement between pairs of MBF and MFR were visualized using Bland-Altman analysis. Ninety-five percent limits of agreement were plotted as mean \pm coefficient of repeatability $(\mathrm{RPC}=1.96 \times \mathrm{SD})$, assuming a gaussian distribution. Three-dimensional scatterplots were used to evaluate the agreement among all 3 methods simultaneously, using a line of best fit (slope and intercept) and the line of identity. The SD of the residual errors (distance from line of best fit) was used to evaluate the disagreement between any method and the average value among all 3 methods. Thus, no single software tool was used as a standard to which the other 2 methods would be compared. Agreement rates were calculated among the programs for classification of scans as abnormal using a stress MBF of less than $2.0 \mathrm{~mL} / \mathrm{min} / \mathrm{g}$ and an MFR of less than 2.0 as reported previously (23). $P$ values of less than 0.05 were considered statistically significant.

\section{RESULTS}

The patient demographics and hemodynamic data are summarized in Table 2. The proportions of male and female subjects were similar (53\% and 47\%); age and body mass index were typical of the patient population referred clinically for assessment of ischemia using myocardial perfusion imaging. The mild increases in heart rate and rate-pressure product from rest to stress were also typical for adenosine and dipyridamole vasodilators.

Global average rest, stress, and flow reserve values were similar using all 3 methods as indicated in Figure 1; 1-way ANOVA indicated no significant bias among methods $(P=$ $0.68,0.14$, and 0.35 , respectively). Average LV flow differences 
TABLE 2

Patient Demographics and Hemodynamics

\begin{tabular}{|c|c|}
\hline Description & Value \\
\hline Number of patients & 90 \\
\hline Age (y) & $66.4 \pm 10.6$ \\
\hline Body mass index $\left(\mathrm{kg} / \mathrm{m}^{2}\right)$ & $30.7 \pm 7.2$ \\
\hline Male sex & $48(53 \%)$ \\
\hline Diabetes mellitus & $33(37 \%)$ \\
\hline Hypertension & $59(66 \%)$ \\
\hline Hyperlipidemia & $60(67 \%)$ \\
\hline Current smoker & 12 (13\%) \\
\hline Family history & $27(30 \%)$ \\
\hline Previous myocardial infarction & $23(26 \%)$ \\
\hline Previous revascularization & $33(37 \%)$ \\
\hline Abnormal perfusion scan & $42(47 \%)$ \\
\hline Rest heart rate (bpm) & $69.6 \pm 13.1$ \\
\hline Rest systolic blood pressure $(\mathrm{mm} \mathrm{Hg})$ & $131.6 \pm 21.3$ \\
\hline Rest rate-pressure product (bpm. $\mathrm{mm} \mathrm{Hg}$ ) & $9,180 \pm 2,409$ \\
\hline Stress heart rate (bpm) & $78.2 \pm 16.9^{\star}$ \\
\hline Stress systolic blood pressure $(\mathrm{mm} \mathrm{Hg})$ & $126.2 \pm 27.3^{*}$ \\
\hline Stress rate-pressure product $(\mathrm{bpm} \cdot \mathrm{mm} \mathrm{Hg})$ & $9,837 \pm 3,370^{\star}$ \\
\hline \multicolumn{2}{|c|}{$\begin{array}{l}{ }^{*} P<0.05 \text { vs. rest. } \\
\text { bpm }=\text { beats per minute. } \\
\text { Values are mean } \pm S D \text {, or number followed by percentage of } \\
\text { tal number of patients in parentheses. }\end{array}$} \\
\hline
\end{tabular}

were no more than $0.04 \mathrm{~mL} / \mathrm{min} / \mathrm{g}$ at rest and no more than $0.28 \mathrm{~mL} / \mathrm{min} / \mathrm{g}$ at stress; Bland-Altman pairwise comparisons between the methods are shown in Figure 2 for MBF and MFR for comparisons between syngoMBF and FlowQuant, FlowQuant and QPET, and QPET and syngoMBF. Small pairwise differences between the methods were noted for global MBF ( $-9 \%$ to $+6 \%)$ and MFR $(-7 \%$ to $+5 \%)$ $(P<0.05)$. The average RPC was 0.26 and 0.29 for the relative MBF and MFR differences, respectively, between programs.

The 3 methods were also compared simultaneously using 3D scatterplots, as shown in Figure 3. The combined correlation was good for MBF $\left(R^{2}>0.97\right.$ for each of the 3 vascular territories, and $R^{2}=0.98$ for global average) and MFR $\left(R^{2}>0.92\right.$ for each territory and $R^{2}=0.95$ for global average). For global MBF, the SE of the regression (SER) was less than $0.14 \mathrm{~mL} / \mathrm{min} / \mathrm{g}$, or $8.4 \%$ of the average MBF across all programs. Regional MBF tended to have a slightly higher SER $(<0.16 \mathrm{~mL} / \mathrm{min} / \mathrm{g})$ across all territories $(P=$ 0.07). For global MFR, the SER was less than 0.23 (9.9\% of mean) across all programs, and regional MFR had a higher SER $(<0.28)$ across all territories $(P=0.02)$. Assuming no bias between any of the 3 programs (i.e., line of identity as best fit), the global average SER remained less than 0.16 $\mathrm{mL} / \mathrm{min} / \mathrm{g}(9.6 \%)$ for rest and stress MBF and less than 0.25 (10.7\%) for MFR as shown in Supplemental Figure 2.

The pairwise agreement between programs ranged from $86 \%$ to $90 \%$ for detection of impaired stress MBF and from $88 \%$ to $91 \%$ for impaired flow reserve as shown in Table 3. All 3 programs identified the same patients with impaired stress MBF of less than $2.0 \mathrm{~mL} / \mathrm{min} / \mathrm{g}$ (or flow reserve $<2.0$ ) in $82 \%$ (or $84 \%$ ) of cases.

\section{DISCUSSION}

To our knowledge, this is the first report comparing different software programs for the routine quantification of $\mathrm{MBF}$ and flow reserve using ${ }^{82} \mathrm{Rb}$ PET imaging. Differences in mean MBF and flow reserve were less than $10 \%$ in general, indicating good agreement among the 3 program implementations of the same tracer kinetic model, using slightly different blood and myocardial sampling methods. These results suggest that the 3 software tools are relatively equivalent for estimation of MBF with ${ }^{82} \mathrm{Rb}$ PET. Good reproducibility of MBF measurements obtained with the 1-tissue-compartment model of ${ }^{82} \mathrm{Rb}$ kinetics also enabled consistent classification ( $\sim 80 \%-90 \%)$ of normal versus abnormal stress MBF and flow reserve. In $10 \%$ and $12 \%$ of patients with pairwise discordance in classification of abnormal flow reserve or stress flow of less than 2.0 (Table 2), the mean differences were small $(0.02 \pm 0.47$ and $0.06 \pm$ 0.21 , respectively), but variance was significantly higher in flow reserve versus stress flow differences among programs $(P<0.001)$. A small number of outliers were evident in the Bland-Altman plots (Fig. 2); only 3 of 540 differences in flow reserve or stress flow $(\sim 0.5 \%)$ were greater than $50 \%$ of the mean value. If a graded classification is used in which normal is $\geq 3.0$, probably normal is $\geq 2.5$, equivocal is $\geq 2.0$, probably abnormal is $<2.0$, and abnormal is $<1.5$, then $98 \%$ of patients fall into the same or an adjacent class. Despite this variability in individual-patient classification, our results suggest that these 3 programs may be used interchangeably in multicenter trials evaluating population outcomes with clinical management directed by absolute blood flow imaging with ${ }^{82} \mathrm{Rb}$ PET.

The present study results are similar to those reported recently by Slomka et al. (5) for ${ }^{13} \mathrm{~N}$-ammonia PET using 1 - or 2-tissue-compartment models, demonstrating a small average

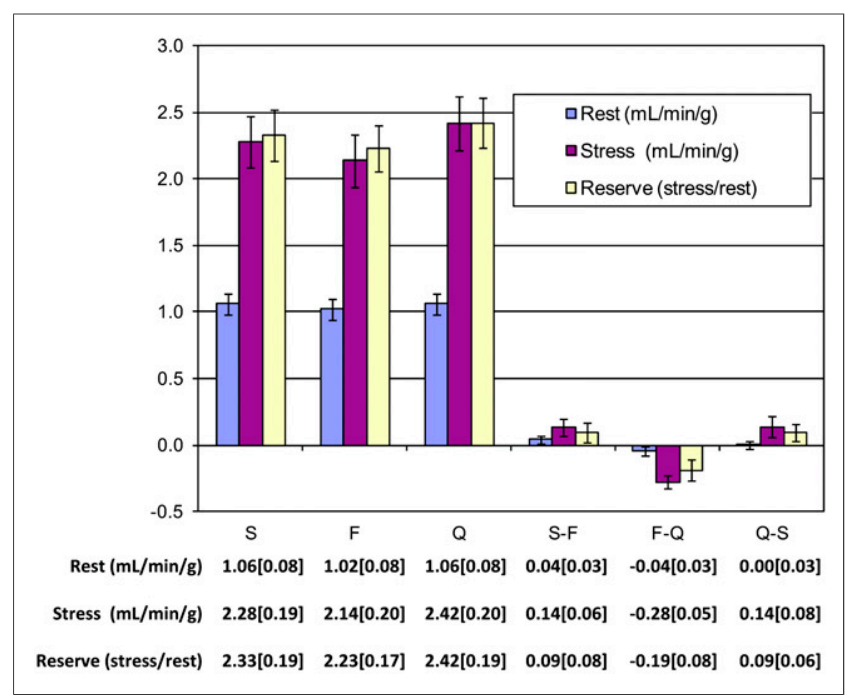

FIGURE 1. Global flow values and $95 \%$ confidence interval measured with syngoMBF (S), FlowQuant (F), and QPET (Q). There were no significant differences among programs for rest $(P=0.68)$, stress $(P=0.14)$, or flow reserve $(P=0.35)$ values. 


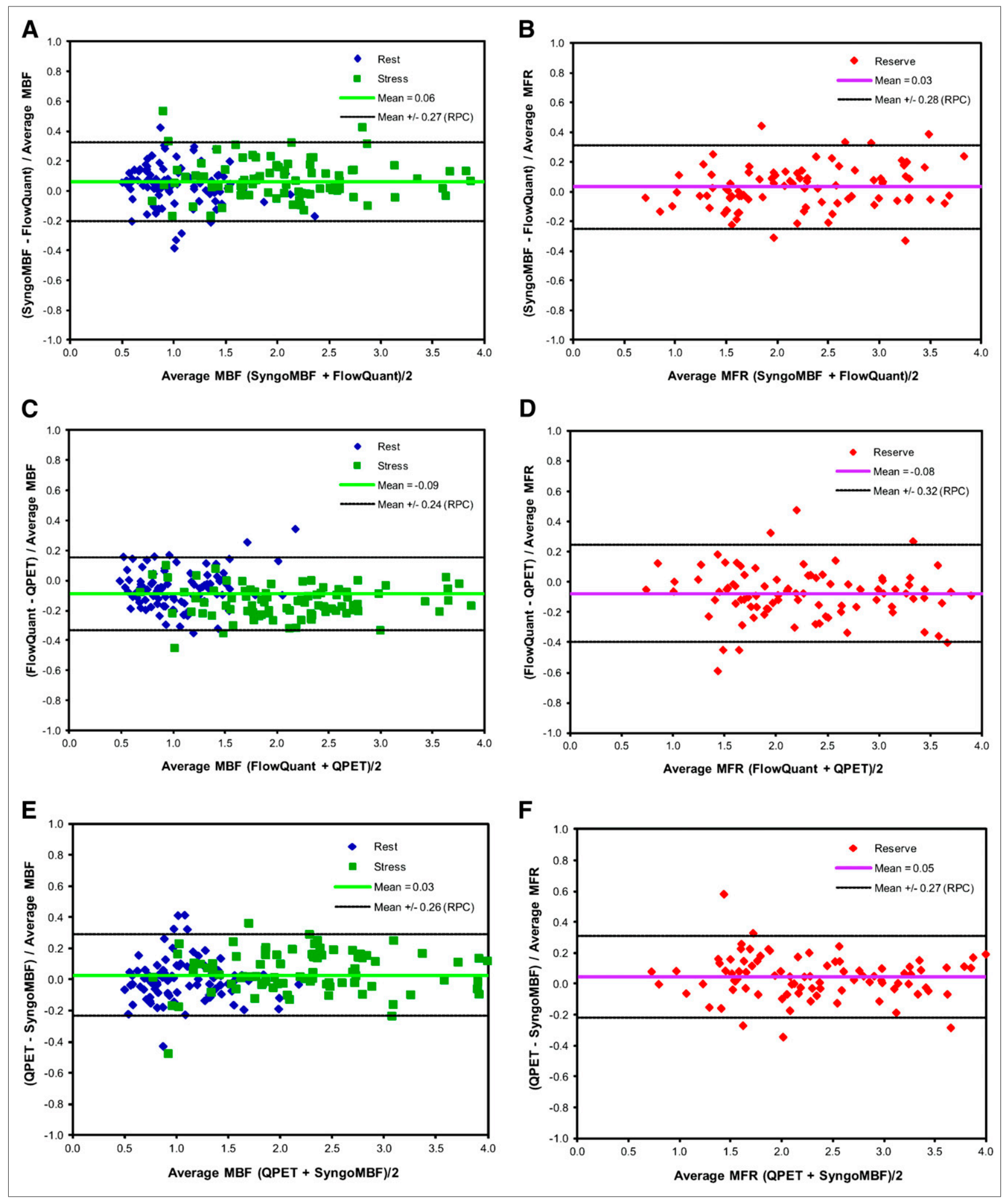

FIGURE 2. Bland-Altman plots of rest and stress MBF differences (A, C, and E) and of MFR differences (reserve; B, D, and F) between syngoMBF and FlowQuant ( $\mathrm{A}$ and $\mathrm{B})$, FlowQuant and QPET (C and $\mathrm{D}$ ), and QPET and syngoMBF (E and F).

bias of $-5 \%$ to $+7 \%$ between MBF measurements and $-7 \%$ to $+8 \%$ for MFR. However, the range of differences among programs appears to be smaller for both MBF and MFR in the present study (SER $<0.14$ and 0.23 ) than in the previous study with ${ }^{13} \mathrm{~N}$-ammonia PET (SER $<0.31$ and 0.74 ). These findings suggest that improved reproducibility in the present 


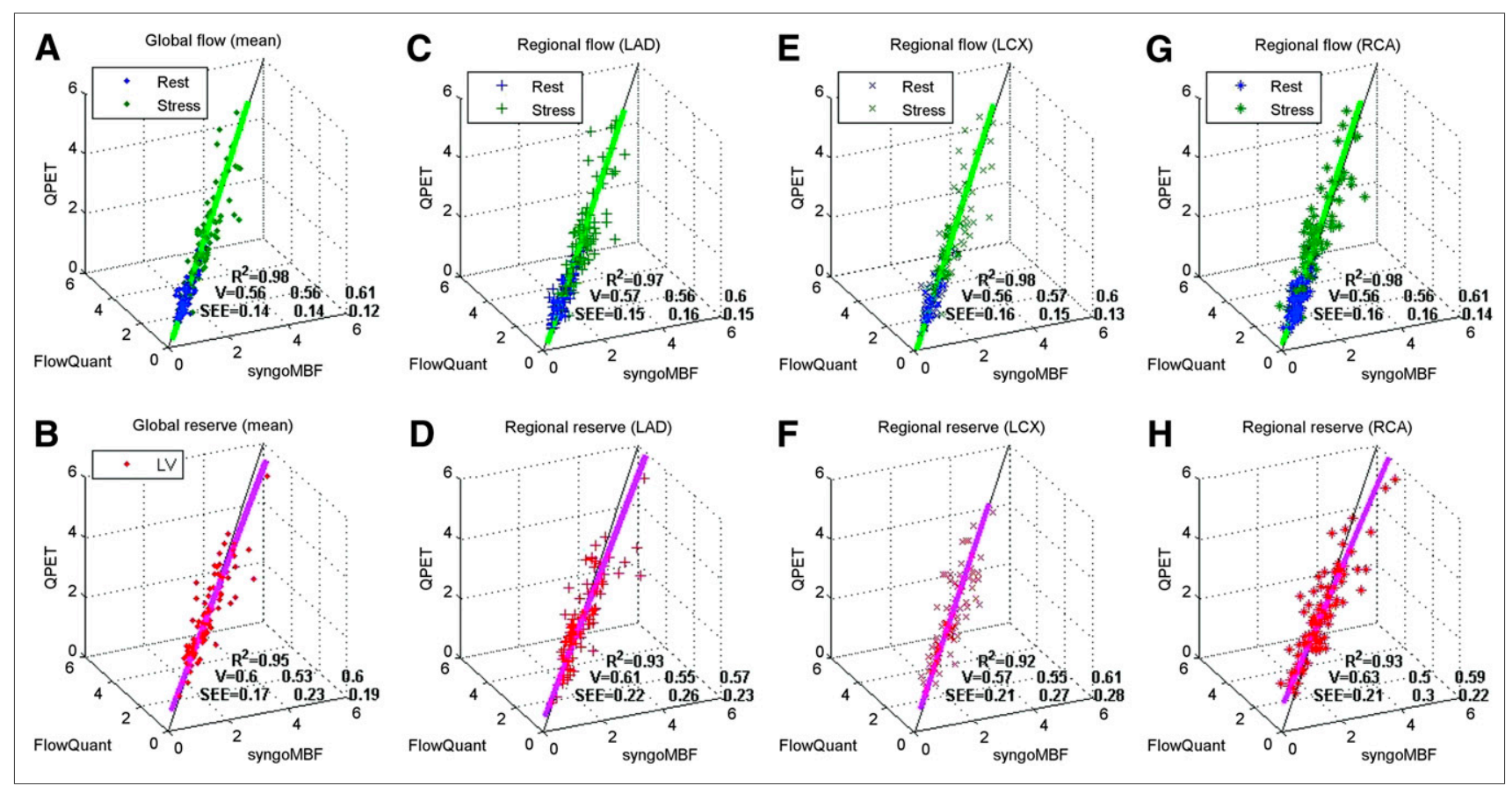

FIGURE 3. 3D scatterplots of syngoMBF vs. FlowQuant vs. QPET values of rest and stress MBF (flow) and MFR (reserve) for global left ventricle $(\mathrm{A}$ and $\mathrm{B})$ and regional vascular territories $(\mathrm{C}-\mathrm{H}) . R^{2}$ is total variance described by $3 \mathrm{D}$ unit basis vector $(\mathrm{V})$ line of best fit. SEE is $\mathrm{SD}$ of residual errors from regression line of best fit for each of the 3 programs.

study may result from the use of the same 1-tissue-compartment model, as opposed to the previous study, in which different tracer kinetic models and software programs were compared. This finding is also reinforced in the study by Khorsand et al. (11), who concluded that program-specific reference databases were required because of significant bias between MBFs obtained with different compartment models used for tracer kinetic analysis of ${ }^{13} \mathrm{~N}$-ammonia PET studies (12).

All 3 software packages provide highly automatic processing with quality control, further promoting common standards for clinical interpretation and research (5-7). However, some small differences between the flow results from the 3 packages remain. The Bland-Altman RPC and SER provide good estimates of the precision of the computed values, allowing a threshold of normal flow to be defined, with some margin of uncertainty for equivocal cases. This may need to be investigated further with a dedicated study protocol involving the generation of reference databases to characterize any potential physiologic dependencies, as well as scanning equipment and protocol variations. For example, whereas the flow reserve values were not significantly different among centers $(P=0.13)$, there were differences in the rest and stress MBFs (2-way ANOVA, $P<0.001$ ), indicating that scanner- or protocol-specific normal cutoff values may be required.

In the present study, the 3 different MBF programs were run by 3 different operators; therefore, part of the observed variability in the results can be attributed to the known effects of intra- and interoperator variability. Using a single program implementation of the 1-tissue-compartment model (FlowQuant), the intra- and interoperator RPC values were reportedly 2\%-8\% for MBF and 8\%-17\% for MFR (7). Similar values of approximately $8 \%-16 \%$ and $16 \%-18 \%$ were reported for MBF and flow reserve, respectively, using syngoMBF (15). Interobserver RPC using QPET was approximately $14 \%$ for MBF and 12\% for flow reserve (5).

This study had several potential limitations. It was limited to only 3D PET/CT scanners; however, this is currently the most prevalent type of PET scanner available commercially. The same patients were not scanned repeatedly on the different 3D PET/CT systems; therefore, differences in MBFs among scanners were not compared. However, the accuracy of flow values should be confirmed for any particular PET system used in clinical practice (24). Because

TABLE 3

Agreement of Global LV Stress MBF and Flow Reserve Interpretation

Measured parameter syngoMBF $=$ FlowQuant FlowQuant $=$ QPET QPET $=$ syngoMBF syngoMBF $=$ FlowQuant $=$ QPET

$\begin{array}{lllll}\text { Stress MBF }<2 \mathrm{~mL} / \mathrm{min} / \mathrm{g} & 90 \%(81 / 90) & 86 \%(77 / 90) & 89 \%(80 / 90) & 82 \%(74 / 90) \\ \text { Stress/rest MFR }<2 & 91 \%(82 / 90) & 88 \%(79 / 90) & 90 \%(81 / 90) & 84 \%(76 / 90) \\ \text { Stress MBF and MFR }<2 & 92 \%(83 / 90) & 89 \%(80 / 90) & 92 \%(83 / 90) & 87 \%(78 / 90)\end{array}$


scan repeatability was not assessed, potential issues with using site- or scanner-specific reference databases for scan assessment will need to be investigated further.

We did not compare the programs separately in each subpopulation from the 3 sites because of the small sample size and the potentially variable clinical referral patterns and patient demographics among sites. All 3 programs processed the data smoothly without limitation to the site of origin, number of frames, scanning duration, or specific scanner restrictions.

There are several sources of variability among the 3 tools, such as different methods of myocardial segmentation and polar map sampling, nonuniform definition of vascular territories, dynamic motion correction, LV input region, and manual interactions. These will lead to some level of variability in the measurements and statistics derived from the computed flow values. Some manual interactions were needed in a minority of cases and were performed separately by 3 different observers at 3 sites. Furthermore, syngoMBF included a motion correction step that was systematically used in this experiment. This study examined the overall variability without attributing it to these separate factors. It is possible that the variation would be smaller if the studies were processed by the same operator using all tools.

We limited the reproducibility comparison in the present study to 3 software tools using the same tracer kinetic model as implemented by each of the groups, but there are other tools available for clinical or research use and further comparisons would be of interest. We did not compare the effectiveness of each tool with respect to detection of the disease according to an external gold standard such as angiography; however, close diagnostic agreement among the tools indicates that such performance should be similar.

\section{CONCLUSION}

The comparison of the 3 software packages (syngoMBF, FlowQuant, and QPET) shows good agreement between $\mathrm{MBF}$ and flow reserve values, with consistent identification of abnormal stress flow and flow reserve in most patients. Differences in program implementation such as motion correction and myocardial sampling parameters, as well as operator variability, likely explain the small residual variations observed.

\section{DISCLOSURE}

The costs of publication of this article were defrayed in part by the payment of page charges. Therefore, and solely to indicate this fact, this article is hereby marked "advertisement" in accordance with 18 USC section 1734. This work was supported by an Imaging in Cardiovascular Therapeutics grant from the Ontario Research Fund (RE02038) and a Molecular Function and Imaging Program grant from the Ontario Heart and Stroke Foundation (PRG6242). Robert deKemp, Ran Klein, and Rob Beanlands are consultants for Jubilant DraxImage. Robert deKemp and Ran Klein receive revenues from the sale of FlowQuant. Piotr Slomka and
Guido Germano receive revenues from the sale of QPET. No other potential conflict of interest relevant to this article was reported.

\section{REFERENCES}

1. Bengel FM, Higuchi T, Javadi MS, Lautamäki R. Cardiac positron emission tomography. J Am Coll Cardiol. 2009;54:1-15.

2. Ziadi MC, Beanlands RS. The clinical utility of assessing myocardial blood flow using positron emission tomography. J Nucl Cardiol. 2010;17:571-581.

3. Bateman TM, Heller GV, McGhie AI, et al. Diagnostic accuracy of rest/stress ECG-gated Rb-82 myocardial perfusion PET: comparison with ECG-gated Tc99m sestamibi SPECT. J Nucl Cardiol. 2006;13:24-33.

4. Klein R, Beanlands RSB, deKemp RA. Quantification of myocardial blood flow and flow reserve: technical aspects. J Nucl Cardiol. 2010;17:555-570.

5. Slomka PJ, Alexanderson E, Jácome R, et al. Comparison of clinical tools for measurements of regional stress and rest myocardial blood flow assessed with ${ }^{13} \mathrm{~N}$-ammonia PET/CT. J Nucl Med. 2012;53:171-181.

6. Sunderland J, Pan XB, Ponto L, Riggert J, Casey M, Declerk J. Interobserver variability of myocardial blood flow and coronary flow reserve with Rb-82 from 3D PET scanners [abstract]. J Nucl Cardiol. 2010;17:738.

7. Klein R, Renaud JM, Ziadi MC, et al. Intra- and inter-operator repeatability of myocardial blood flow and myocardial flow reserve measurements using rubidium-82 PET and a highly automated analysis program. J Nucl Cardiol. 2010; 17:600-616.

8. El Fakhri G, Kardan A, Sitek A, et al. Reproducibility and accuracy of quantitative myocardial blood flow assessment with ${ }^{82} \mathrm{Rb}$ PET: comparison with ${ }^{13} \mathrm{~N}$ ammonia PET. J Nucl Med. 2009;50:1062-1071.

9. Kajander S, Joutsiniemi E, Saraste M, et al. Cardiac positron emission tomography/computed tomography imaging accurately detects anatomically and functionally significant coronary artery disease. Circulation. 2010;122:603-613.

10. Lortie M, Beanlands RS, Yoshinaga K, Klein R, Dasilva JN, deKemp RA. Quantification of myocardial blood flow with ${ }^{82} \mathrm{Rb}$ dynamic PET imaging. Eur J Nucl Med Mol Imaging. 2007;34:1765-1774.

11. Khorsand A, Graf S, Pirich C, et al. Assessment of myocardial perfusion by dynamic N-13 ammonia PET imaging: comparison of 2 tracer kinetic models. J Nucl Cardiol. 2005;12:410-417.

12. Choi Y, Huang SC, Hawkins RA, et al. Quantification of myocardial blood flow using ${ }^{13} \mathrm{~N}$-ammonia and PET: comparison of tracer models. J Nucl Med. 1999;40:1045-1055.

13. Cerqueira MD, Weissman NJ, Dilsizian V, et al. Standardized myocardial segmentation and nomenclature for tomographic imaging of the heart: a statement for healthcare professionals from the Cardiac Imaging Committee of the Council on Clinical Cardiology of the American Heart Association. Circulation. 2002; 105:539-542.

14. Klein R, Adler A, Beanlands RS, deKemp RA. Precision-controlled elution of a ${ }^{82} \mathrm{Sr} /{ }^{82} \mathrm{Rb}$ generator for cardiac perfusion imaging with positron emission tomography. Phys Med Biol. 2007;52:659-673.

15. Pan XB, Declerck J. White Paper: Validation syngo.PET Myocardial Blood Flow. Hoffman Estates, IL: Siemens Medical Solutions; 2011:1-17.

16. Pan XB, Schindler TH, Ratib O, Nekolla S, Declerck J. Effect of reorientation on myocardial blood flow estimation from dynamic ${ }^{13} \mathrm{NH}_{3}$ PET imaging. IEEE Nucl Sci Symp Med Imaging Conf Rec. 2009;1:3715-3717.

17. Bond S, Pan XB, Declerck J. A study of consistency of myocardial blood flow calculation using motion correction in dynamic PET [abstract]. J Nucl Med. 2010;51(suppl 2):253P.

18. Nakazato R, Berman DS, Dey D, et al. Automated quantitative Rb-82 3D PET/ CT myocardial perfusion imaging: normal limits and correlation with invasive coronary angiography. J Nucl Cardiol. 2012;19:265-276.

19. Germano G, Kavanagh PB, Chen J, et al. Operator-less processing of myocardial perfusion SPECT studies. J Nucl Med. 1995;36:2127-2132.

20. Coxson PG, Huesman RH, Borland L. Consequences of using a simplified kinetic model for dynamic PET data. J Nucl Med. 1997;38:660-667.

21. Renkin EM. Transport of potassium- 42 from blood to tissue isolated mammalian skeletal muscles. Am J Physiol. 1959;197:1205-1210.

22. Crone C. Permeability of capillaries in various organs as determined by use of the indicator diffusion method. Acta Physiol Scand. 1963;58:292-305.

23. Ziadi MC, deKemp RA, Williams KA, et al. Impaired myocardial flow reserve on rubidium-82 positron emission tomography imaging predicts adverse outcomes in patients assessed for myocardial ischemia. J Am Coll Cardiol. 2011; 58:740-748.

24. deKemp RA, Klein R, Renaud J, et al. 3D listmode cardiac PET for simultaneous quantification of myocardial blood flow and ventricular function. IEEE Nucl Sci Symp Med Imaging Conf Rec. 2008:5215-5218. 